University of Nebraska - Lincoln

DigitalCommons@University of Nebraska - Lincoln

2010

\title{
Yeasts associated with nectarines and their potential for biological control of brown rot
}

\author{
W. J. Janisiewicz \\ USDA-ARS, wojciech.janisiewicz@ars.usda.gov \\ C. P. Kurtzman \\ USDA-ARS
}

J. S. Buyer

USDA-ARS

Follow this and additional works at: https://digitalcommons.unl.edu/usdaarsfacpub

Part of the Agricultural Science Commons

Janisiewicz, W. J.; Kurtzman, C. P.; and Buyer, J. S., "Yeasts associated with nectarines and their potential for biological control of brown rot" (2010). Publications from USDA-ARS / UNL Faculty. 563.

https://digitalcommons.unl.edu/usdaarsfacpub/563

This Article is brought to you for free and open access by the U.S. Department of Agriculture: Agricultural Research Service, Lincoln, Nebraska at DigitalCommons@University of Nebraska - Lincoln. It has been accepted for inclusion in Publications from USDA-ARS / UNL Faculty by an authorized administrator of DigitalCommons@University of Nebraska - Lincoln. 


\title{
Yeasts associated with nectarines and their potential for biological control of brown $\operatorname{rot}^{\dagger}$
}

\author{
W. J. Janisiewicz I*, C. P. Kurtzman'² and J. S. Buyer ${ }^{3}$ \\ 'Appalachian Fruit Research Station, ARS-USDA, Kearneysville, WV, USA \\ ${ }^{2}$ National Center for Agricultural Utilization Research, ARS-USDA, Peoria, IL, USA \\ ${ }^{3}$ Sustainable Agricultural Systems Laboratory, Henry A. Wallace Beltsville Agricultural Research Center, ARS-USDA, Beltsville, MD, USA
}

*Correspondence to:
W. J. Janisiewicz, Appalachian
Fruit Research Station,
ARS-USDA, 22 I 7 Wiltshire Road,
Kearneysville, WV 25430, USA.
E-mail: wojciech.janisiewicz@
ars.usda.gov
${ }^{\dagger}$ Mention of trade names or
commercial products in this
paper is solely for the purpose of
providing specific information and
does not imply recommendation
or endorsement by the US
Department of Agriculture.

Received: 2 October 2009 Accepted: 17 January 2010

\begin{abstract}
Resident fruit microflora has been the source of biocontrol agents for the control of postharvest decay of fruits and the active ingredient in commercialized biocontrol products. With the exception of grapes and apples, information on the resident microflora of other fruits is only fragmentary, but greater knowledge in this area can be very helpful in developing biocontrol strategies. We characterized the yeast microflora of nectarines ('Croce del Sud') from the early stages of fruit development until harvest. The fruit samples were collected from trees in an unmanaged orchard. The resident fruit microflora was separated from the occasionally deposited microorganisms by discarding initial fruit washings before the final wash, followed by sonication and plating on NYDA medium. The isolated yeasts were identified by BIOLOG and by sequencing the D1/D2 domain of a large subunit of the rRNA gene and, where available, the ITS sequence. BIOLOG identified 19 and the genetic analysis 23 species of yeasts. Although the identification by these two systems was not always the same, the predominant yeasts were Rhodotorula spp., Sporodiobolus spp., Cryptococcus spp., Pichia spp., Candida spp. and yeast-like Aureobasidium pullulans. Several of the taxa appear to represent new species. The preliminary biocontrol tests against brown rot of nectarine fruit caused by Monilinia fructicola indicates significant decay control potential of some of the identified yeast species, namely Cryptococcus magnus, Cryptococcus sp. nov., Sporidiobolus pararoseus, A. pullulans and Rhodotorula sp. nov. Copyright $\odot 2010$ John Wiley \& Sons, Ltd.
\end{abstract}

Keywords: yeast communities; fruit microflora; postharvest biocontrol; Monilinia fructicola

\section{Introduction}

Natural fruit surface microflora includes yeasts, bacteria and filamentous fungi. The growth of these microorganisms is supported by nutrients leaking from the fruit, as well as external sources, such as pollen deposits, a variety of organic debris and honeydew (Blakeman, 1985). These sources provide the food base for saprophytic microorganisms and can be utilized by parasities as well, before establishing parasitic interaction with the host. As the season progresses, the composition of the fruit surface microflora changes (Janisiewicz and Buyer, 2010). This may be due to flux in the nutritional status of the fruit, such as differences in abundance of carbon and nitrogen compounds (sugar and amino acids) and tissue $\mathrm{pH}$. Under favourable climatic conditions, such as moderate temperatures and adequate moisture, microorganisms may flourish on the fruit surface. In biological control of postharvest diseases (BCPD) of fruit, the fruit microflora has been the main source of antagonists (Chand-Goyal and Spotts, 1996; Droby et al., 1999; Janisiewicz, 1987; Wilson et al., 1993; Usall 
et al., 2000), some of which have been commercialized (Janisiewicz and Korsten, 2002). Antagonists selected from the resident fruit microflora may have ecological advantages over antagonists originating from microflora casually deposited on the fruit or those residing mainly in the soil. They may also be more acceptable as a postharvest fruit treatment to the public and regulatory agencies, since they have been consumed with fruit for millennia without known adverse effects.

In comparison to other plant parts, relatively little is known about the microbial ecology of fruit surfaces. Most of the work was done on grape and apple, often in relation to vine and apple cider production (Beech, 1993; Clark et al., 1954; Davenport 1976a, 1976b; Waida et al., 1983; Williams et al., 1956) and most recently on pome and citrus fruits in relation to biological control of fruit decays (Droby et al. 1999; Chand-Goyal and Spotts, 1996; Janisiewicz, 1987; Janisiewicz et al., 2001). Knowledge of microbial ecology of other fruits, including stone fruits, is often limited to reports on the presence of the individual microorganisms at a given time, without any attempt to determine their residential status or place in the microbial succession (Buhagiar and Barnett, 1971; Dennis, 1976; Sasaki and Yoshida, 1959; Stallarova, 1982). The natural microflora of plums and nectarines has not been characterized and explored for their biocontrol potential, and information about these microflora is limited to a description of a few yeast species on plums and cherries (Stollarova, 1982, 1984).

The main objective of this study was to describe the resident yeast microflora of intact nectarine fruit from the early stages of fruit development to maturity, and to determine their antagonistic potential against Monilinia fructicola, the causal agent of brown rot, which is a very destructive postharvest disease of stone fruits.

\section{Materials and methods}

\section{Orchard and fruit sampling}

Nectarine trees 'Croce del Sud' were grown in an unmanaged orchard at the Appalachian Fruit Research Station in Kearneysville, WV. The trees were 7 years old during the first year of isolation. Fruit samples for isolations were collected aseptically from five trees, four times during fruit development from early stages until maturity, in two consecutive years (2006 and 2007).

\section{Microbe isolation}

The fruit samples were washed in $500 \mathrm{ml}$ beakers with phosphate buffer $(0.05 \mathrm{M}$, at $\mathrm{pH} 6.8)$ by shaking on a rotary shaker for $3 \mathrm{~min}$ at $75 \mathrm{rpm}$. The washings were discarded, and fresh buffer was added to the beakers. The fruit was sonicated using a Branson 1510 sonication bath (Branson Ultrasonic Corp., Eagle Road, Danbury, CT, USA) for $1 \mathrm{~min}$ and washed again by shaking for $15 \mathrm{~min}$ at $75 \mathrm{rpm}$. These washings were dilutionplated on nutrient yeast dextrose agar (NYDA) medium (Difco, Becton-Dickinson, Sparks, MD, USA). The plates were incubated at $26^{\circ} \mathrm{C}$ for up to 7 days and developing colonies were isolated randomly and based on different phenotypic characteristics. The plates were re-examined after 2 weeks of incubation at $4{ }^{\circ} \mathrm{C}$ and additional isolations were made. The colonies were purified by triple restreaking of single colonies and preserved in $15 \%$ glycerol at $-80^{\circ} \mathrm{C}$. The code for the isolates reflected nectarine $(\mathrm{Ne})$ as the source of isolation, the year of isolation 6 or 7 (for 2006 or 2007), the time of isolation [T1, 6 weeks before harvest (wbh); T2, 4 wbh; T3, 2 wbh; T4, harvest), the tree sample (S) and the isolate (I) number. The AFRS-Kearneysville and NRRL Collection (Peoria, IL, USA) codes are listed in Table 2.

\section{Isolates identification}

Purified isolates were differentiated as to bacteria, yeast, and filamentous fungi. The yeasts were identified using the BIOLOG ML 4.2 system (Biolog Inc., Hayward, CA, USA) and genetically, using the sequences of the D1/D2 domain of the large subunit $(L S U)$ rRNA gene and the ITS sequence according to procedures described by Kurtzman and Robnett (1998, 2003).

\section{Preparation of pathogen and yeast inocula}

The pathogen, Monilinia fructicola (isolate Mf7) was isolated from a decayed peach and was maintained on peach agar medium (PA) under constant light at $22^{\circ} \mathrm{C}$. The PA medium contained $900 \mathrm{~g} / \mathrm{l}$ canned peach halves in heavy syrup, which were washed three times in sterile distilled water, 
blended in a blender and $22 \mathrm{~g} / \mathrm{l}$ agar was added. Conidia were collected from 10-14 day-old sporulating cultures with a vacuum spore collector, suspended in $0.05 \%$ Tween 20 , sonicated, vortexed and adjusted with water to a concentration of $10^{5}$ conidia/ml, using a haemocytometer. Then, two 10fold dilutions were made to obtain two additional concentrations of $10^{4}$ and $10^{3}$ conidia/ml, which were also used for fruit inoculation.

The yeast cultures were activated from stock cultures by transferring to plates with NYDA medium. After growing to easily visible culture, they were transferred again to NYDA medium, grown overnight, and the yeast suspensions were prepared by suspending the culture in sterile tap water and adjusting the concentration to $50 \%$ transmittance at $420 \mathrm{~nm}$.

\section{Biocontrol tests}

Throughput screening of the yeast isolates was conducted on nectarine fruits. Harvested fruits were washed, randomized and placed on fruit pack trays in plastic boxes. The fruits were wounded in the middle with a cylindrical tool, $3 \mathrm{~mm}$ in diameter and $3 \mathrm{~mm}$ deep, and the cut tissue was removed; $25 \mu \mathrm{l}$ of the aqueous antagonist suspension was placed in each wound, and after approximately $1.5 \mathrm{~h}$ the wounds were inoculated with $25 \mu \mathrm{l}$ of a conidial suspension of $M$. fructicola at concentrations of $10^{3}, 10^{4}$ or $10^{5}$ conidia/ml. The fruits were incubated at $22^{\circ} \mathrm{C}$ for 3 days and the lesion size was measured perpendicular to the stem axis. Each isolate was tested on nine fruits, three for each concentration of the pathogen.

\section{Data analysis}

BIOLOG data from individual trees and for samples collected during the season were combined and subjected to BIOLOG cluster analysis (program included in the BIOLOG MicroLog ${ }^{\mathrm{TM}} 3$ System, Version 4.20.05, Biolog Inc., Hayward, CA, USA), and dendrograms expressing units of taxonomic distance were generated (see dendrograms at: http://www.ars.usda.gov/pandp/docs.htm?docid =17505). To compare the two identification methods, results from the genetic identification were listed, along with the BIOLOG identification, on the dendrograms. ITS sequences were aligned in CLC Sequence Viewer 6.1 (http://www.clcbio. com). A distance matrix was constructed from the aligned sequences using the Phylip program dnadist (http://evolution.genetics.washington.edu/ phylip.html). The distance matrix was analysed and graphed by non-metric multidimensional scaling, using proc MDS in SAS (SAS Institute, Cary, NC, USA). ANOVA (proc GLM, SAS) was used to determine whether the coordinates generated by non-metric multidimensional scaling were significantly different at different sampling times. Species abundance tables (species or genus vs. sampling time) were constructed and statistically tested using proc FREQ (SAS). Fisher's exact test was conducted using the Monte Carlo estimation.

\section{Results and discussion}

Our results indicate that the genetic identification is more consistent and reliable than BIOLOG identification so all of our references to the identity of the isolates are based on genetic identification. Nineteen species were identified by BIOLOG (see data at: http://www.ars.usda.gov/pandp/docs.htm? docid=17505) and 23 species and 17 genera were identified by genetic methods (Tables 1,2 ). The species identified by BIOLOG were often different from those identified genetically. Nevertheless, the BIOLOG grouping often reflected genetic identification, indicating a very limited library in the BIOLOG system. Cluster analysis of the BIOLOG data resulted in 11 major clusters that were complementary with genetic rather than BIOLOG identification. For example, one cluster had 17 isolates which represented only two species using genetic identification, while BIOLOG identified four species and only half of the isolates were identified.

Cryptococcus spp., Aureobasidium sp., Rhodotorula spp. and Sporidiobolus sp. were isolated throughout fruit development and together constituted $84.72 \%$ of all isolations (Table 1). Other species, such as Erythrobasidium, Pseudeurotium or Tremella were isolated during the early stage of fruit development, while Hanseniaspora, Candida, Acremonium, Metschnikowia, Pichia, Sporobolomyces and Zygosaccharomyces were isolated mainly from mature fruits. Some isolates were identified by the genetic methods only to genus, and their species identity awaits further investigation. Several of the taxa appear to represent new species. 
Table I. Frequency analysis of the occurrence of yeast genera at various sampling times during a 2 year period based on the DNA ITS sequence distance data analysis

\begin{tabular}{|c|c|c|c|c|c|}
\hline \multirow[b]{2}{*}{ Genus } & \multicolumn{4}{|c|}{ Time of sampling } & \multirow[b]{2}{*}{ Tota } \\
\hline & $\mathbf{T I}^{\prime}$ & T2 & T3 & T4 & \\
\hline Acremonium & $\begin{array}{l}0^{2} \\
0.00^{3} \\
0.00^{4} \\
0.00^{5}\end{array}$ & $\begin{array}{l}0 \\
0.00 \\
0.00 \\
0.00\end{array}$ & $\begin{array}{l}0 \\
0.00 \\
0.00 \\
0.00\end{array}$ & $\begin{array}{c}1 \\
0.55 \\
100.00 \\
2.94\end{array}$ & $\begin{array}{l}\text { I } \\
0.55\end{array}$ \\
\hline Aureobasidium & $\begin{array}{c}12 \\
6.59 \\
27.91 \\
30.00\end{array}$ & $\begin{array}{c}13 \\
7.14 \\
30.23 \\
22.03\end{array}$ & $\begin{array}{c}11 \\
6.04 \\
25.58 \\
22.45\end{array}$ & $\begin{array}{c}7 \\
3.85 \\
16.28 \\
20.59\end{array}$ & $\begin{array}{l}43 \\
23.63\end{array}$ \\
\hline Auriculibuller & $\begin{array}{c}2 \\
1.10 \\
66.7 \\
5.00\end{array}$ & $\begin{array}{l}0 \\
0.00 \\
0.00 \\
0.00\end{array}$ & $\begin{array}{r}1 \\
0.55 \\
33.33 \\
2.04\end{array}$ & $\begin{array}{l}0 \\
0.00 \\
0.00 \\
0.00\end{array}$ & $\begin{array}{l}3 \\
1.65\end{array}$ \\
\hline Candida & $\begin{array}{l}0 \\
0.00 \\
0.00 \\
0.00\end{array}$ & $\begin{array}{l}0 \\
0.00 \\
0.00 \\
0.00\end{array}$ & $\begin{array}{l}0 \\
0.00 \\
0.00 \\
0.00\end{array}$ & $\begin{array}{c}2 \\
1.10 \\
100.00 \\
5.88\end{array}$ & $\begin{array}{l}2 \\
1.10\end{array}$ \\
\hline Cryptococcus & $\begin{array}{c}9 \\
4.95 \\
18.75 \\
22.5\end{array}$ & $\begin{array}{c}17 \\
9.34 \\
35.42 \\
28.81\end{array}$ & $\begin{array}{c}14 \\
7.69 \\
29.17 \\
28.57\end{array}$ & $\begin{array}{c}8 \\
4.40 \\
16.67 \\
23.53\end{array}$ & $\begin{array}{l}48 \\
26.37\end{array}$ \\
\hline Dothichiza & $\begin{array}{l}0 \\
0.00 \\
0.00 \\
0.00\end{array}$ & $\begin{array}{l}0 \\
0.00 \\
0.00 \\
0.00\end{array}$ & $\begin{array}{c}1 \\
0.55 \\
100.00 \\
2.04\end{array}$ & $\begin{array}{l}0 \\
0.00 \\
0.00 \\
0.00\end{array}$ & $\begin{array}{l}\text { I } \\
0.55\end{array}$ \\
\hline Erythrobasidium & $\begin{array}{c}1 \\
0.55 \\
16.67 \\
2.5\end{array}$ & $\begin{array}{r}5 \\
2.75 \\
83.33 \\
8.47\end{array}$ & $\begin{array}{l}0 \\
0.00 \\
0.00 \\
0.00\end{array}$ & $\begin{array}{l}0 \\
0.00 \\
0.00 \\
0.00\end{array}$ & $\begin{array}{l}6 \\
3.30\end{array}$ \\
\hline Hanseniaspora & $\begin{array}{l}0 \\
0.00 \\
0.00 \\
0.00\end{array}$ & $\begin{array}{l}0 \\
0.00 \\
0.00 \\
0.00\end{array}$ & $\begin{array}{l}0 \\
0.00 \\
0.00 \\
0.00\end{array}$ & $\begin{array}{r}1 \\
0.55 \\
100.00 \\
2.94\end{array}$ & $\begin{array}{l}\text { l } \\
0.55\end{array}$ \\
\hline Metschnikowia & $\begin{array}{l}0 \\
0.00 \\
0.00 \\
0.00\end{array}$ & $\begin{array}{c}\text { I } \\
0.55 \\
50.00 \\
1.69\end{array}$ & $\begin{array}{l}0 \\
0.00 \\
0.00 \\
0.00\end{array}$ & $\begin{array}{r}1 \\
0.55 \\
50.00 \\
2.94\end{array}$ & $\begin{array}{l}2 \\
1.10\end{array}$ \\
\hline Pichia & $\begin{array}{l}0 \\
0.00 \\
0.00 \\
0.00\end{array}$ & $\begin{array}{l}0 \\
0.00 \\
0.00 \\
0.00\end{array}$ & $\begin{array}{l}0 \\
0.00 \\
0.00 \\
0.00\end{array}$ & $\begin{array}{c}1 \\
0.55 \\
100.00 \\
2.94\end{array}$ & $\begin{array}{l}\text { I } \\
0.55\end{array}$ \\
\hline Pseudoeurotium & $\begin{array}{c}1 \\
0.55 \\
100.00 \\
2.5\end{array}$ & $\begin{array}{l}0 \\
0.00 \\
0.00 \\
0.00\end{array}$ & $\begin{array}{l}0 \\
0.00 \\
0.00 \\
0.00\end{array}$ & $\begin{array}{l}0 \\
0.00 \\
0.00 \\
0.00\end{array}$ & $\begin{array}{l}1 \\
0.55\end{array}$ \\
\hline Rhodotorula & $\begin{array}{c}11 \\
6.04 \\
26.83 \\
27.50\end{array}$ & $\begin{array}{c}14 \\
7.69 \\
34.15 \\
23.73\end{array}$ & $\begin{array}{c}11 \\
6.04 \\
26.83 \\
22.45\end{array}$ & $\begin{array}{c}5 \\
2.75 \\
12.20 \\
|4.7|\end{array}$ & $\begin{array}{l}41 \\
22.53\end{array}$ \\
\hline
\end{tabular}

Copyright (c) 2010 John Wiley \& Sons, Ltd.
Table I. Continued

\begin{tabular}{|c|c|c|c|c|c|}
\hline \multirow[b]{2}{*}{ Genus } & \multicolumn{4}{|c|}{ Time of sampling } & \multirow[b]{2}{*}{ Total } \\
\hline & TI & T2 & T3 & T4 & \\
\hline oridiobolus & $\begin{array}{c}3 \\
1.65 \\
12.5 \\
7.5\end{array}$ & $\begin{array}{c}7 \\
3.85 \\
29.17 \\
11.86\end{array}$ & $\begin{array}{c}8 \\
4.40 \\
33.33 \\
16.33\end{array}$ & $\begin{array}{c}6 \\
3.30 \\
25.00 \\
17.65\end{array}$ & $\begin{array}{l}24 \\
13.19\end{array}$ \\
\hline porobolomyces & $\begin{array}{l}0 \\
0.00 \\
0.00 \\
0.00\end{array}$ & $\begin{array}{l}0 \\
0.00 \\
0.00 \mid \\
0.00\end{array}$ & $\begin{array}{c}2 \\
1.10 \\
100.00 \\
4.08\end{array}$ & $\begin{array}{l}0 \\
0.00 \\
0.00 \\
0.00\end{array}$ & $\begin{array}{l}2 \\
1.10\end{array}$ \\
\hline Taphrina & $\begin{array}{c}1 \\
0.55 \\
25.00 \\
2.50\end{array}$ & $\begin{array}{c}1 \\
0.55 \\
25.00 \\
2.50\end{array}$ & $\begin{array}{c}\text { । } \\
0.55 \\
25.00 \\
2.50\end{array}$ & $\begin{array}{c}\text { I } \\
0.55 \\
25.00 \\
2.50\end{array}$ & $\begin{array}{l}4 \\
2.20\end{array}$ \\
\hline Tremella & $\begin{array}{l}0 \\
0.00 \\
0.00 \\
0.00\end{array}$ & $\begin{array}{c}1 \\
0.55 \\
100.00 \\
1.69\end{array}$ & $\begin{array}{l}0 \\
0.00 \\
0.00 \\
0.00\end{array}$ & $\begin{array}{l}0 \\
0.00 \\
0.00 \\
0.00\end{array}$ & $\begin{array}{l}\text { I } \\
0.55\end{array}$ \\
\hline Zygosaccharomyces & $\begin{array}{l}0 \\
0.00 \\
0.00 \\
0.00\end{array}$ & $\begin{array}{l}0 \\
0.00 \\
0.00 \\
0.00\end{array}$ & $\begin{array}{l}0 \\
0.00 \\
0.00 \\
0.00\end{array}$ & \begin{tabular}{r}
\multicolumn{1}{c}{1} \\
0.55 \\
100.00 \\
2.94
\end{tabular} & 0.55 \\
\hline $\begin{array}{l}\text { Number of isolates } \\
\text { Percentage of total isolates }\end{array}$ & $\begin{array}{l}40 \\
21.98\end{array}$ & $\begin{array}{l}59 \\
32.42\end{array}$ & $\begin{array}{l}49 \\
26.92\end{array}$ & $\begin{array}{l}34 \\
18.68\end{array}$ & $\begin{array}{l}182 \\
100.00\end{array}$ \\
\hline $\begin{array}{l}\text { I Time of sampling: TI, } 6 \text { we } \\
2 \text { wbh; T4, harvest. } \\
2 \text { Frequency of occurrence } \\
\text { sampling time. } \\
{ }^{3} \text { Percentage of a genus in } r \\
\text { sampling times. } \\
{ }^{4} \text { Percentage of a genus in } \\
\text { times. } \\
5 \text { Percentage of a genus in }\end{array}$ & $\begin{array}{l}\text { (numb } \\
\text { relation } \\
\text { relatio } \\
\text { relatio }\end{array}$ & $\begin{array}{l}\text { of isola } \\
\text { all isolat } \\
\text { this ge } \\
\text { all gen }\end{array}$ & $\begin{array}{l}\text { ates) of } \\
\text { enus at }\end{array}$ & $\begin{array}{l}\text { he gene } \\
\text { genera } \\
\text { arious sa }\end{array}$ & $\begin{array}{l}\text { oh; T3, } \\
\text { om at a } \\
\text { mpling } \\
\text { mpling }\end{array}$ \\
\hline
\end{tabular}

Non-metric multidimensional scaling was used to graphically represent the yeast community (Figure 1a). The final badness of fit criterion, equivalent to Kruskal's stress formula 1, was 0.120 , which is considered satisfactory for ecological data (McCune and Grace, 2002). While the communities in each of the first three sampling times were not differentiated from each other by this analysis, the community of the fourth sampling time partially separated from the other three (Figure 1b). The separation of sampling 4 from the first three samplings was statistically significant for dimension 2 in $2006(p=0.0001)$ but not in 2007. The effect of sampling time on the distribution of isolates within genera was further analysed by frequency analysis (Table 1). The probability of no

Yeast 2010; 27: 389-398. DOI: 10.1002/yea 

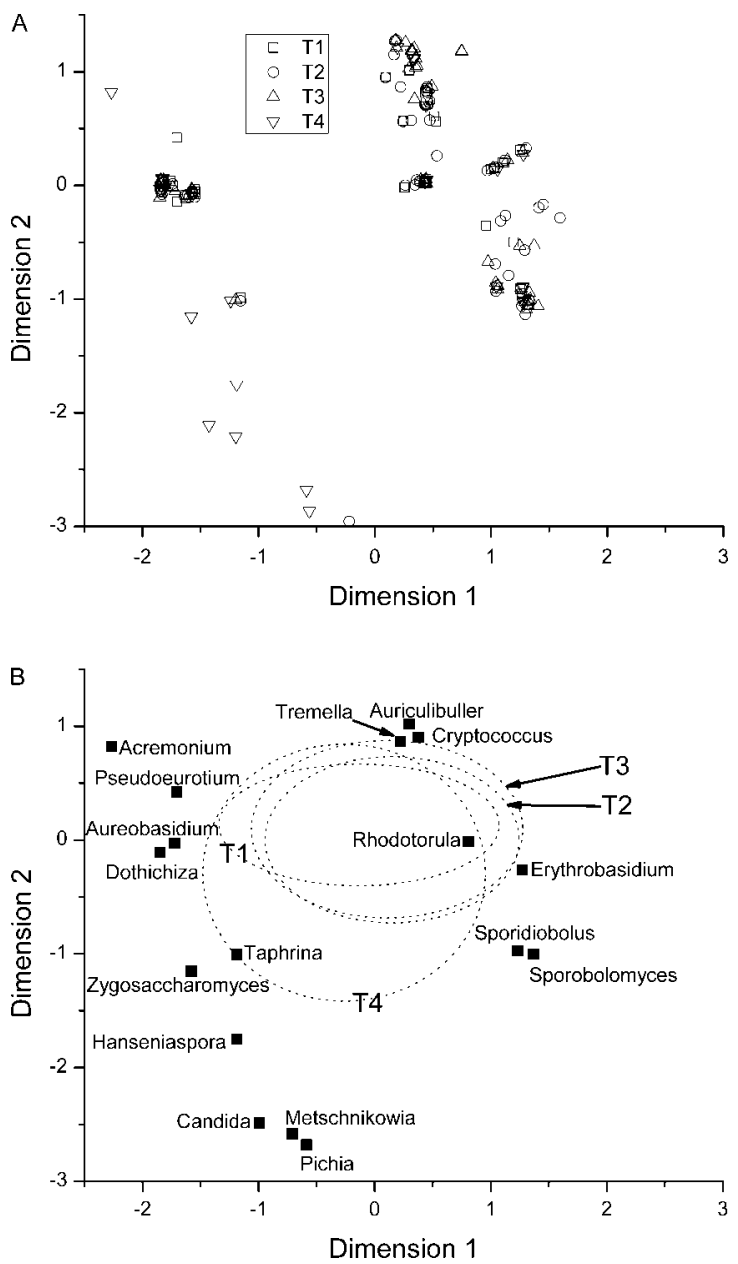

Figure I. Non-metric multidimensional scaling of distance matrix of ITS sequence data of yeasts. (A) All samples from both years were plotted; squares, TI; circles, T2; triangles, T3; inverted triangles, T4. (B) The means for each genus were plotted as points, while the means and standard deviations for each sampling time were plotted as ellipses

association between genus and time was $1 \times 10^{-20}$, indicating a statistically significant effect of time on genus.

In contrast to yeast, bacterial communities isolated at the same time and from the same trees as the yeast in this study were significantly different from each other at each time point (Janisiewicz and Buyer, 2010). While the analytical and statistical methods used for bacteria and yeasts were different, it still appears that yeast succession at the species and genus levels was much less pronounced than bacterial succession. This may result from better adaptation to the changing fruit environment by the few yeasts, which was demonstrated by their dominance throughout the growing season.

Three out of four of the most predominant yeasts, Cryptococcus spp., Aureobasidium sp. and Rhodotorula spp., have been reported to be effective biocontrol agents against a number of postharvest diseases of various fruits (Calvente, 2001; Ippolito and Nigro, 2000; Leibinger et al., 1997; Lima et al. 1998; Roberts, 1990; Zhang et al., 2008). Aureobasidium was also reported to control field diseases (Dimakopoulus et al., 2008; Kunz, 2004; Lima et al. 1997). This may result from the ability to colonize fruits throughout the growing season. This is in contrast to yeasts isolated from fruit at the most mature stage, such as Zygosaccharomyces bailii or Hanseniaspora uvarum, which could be explored for postharvest protection. However, their usefulness must be carefully considered, as they may be involved in the spoilage of fruit juices. In research on developing biocontrol of field diseases of stone fruit, it may also be advantageous to focus on Cryptococcus spp., Rhodotorula spp. and Sporidiobolus sp. as they also colonize fruit throughout the growing season.

Results from the biocontrol test on controlling infections originating from wounds after harvest indicate a significant potential of some of these yeasts for controlling infections originating from wounds (Table 3). There was a wide range of effectiveness, even between isolates within a species. The most effective yeasts were Cryptococcus magnus, Cryptococcus sp. nov., Aureobasidium pullulans, Rhodotorural sp. nov. and Sporidiobolus papraroseus. However, more research is needed to determine their usefulness for practical applications against brown rot.

This is the first report describing residential populations of yeasts on nectarine fruit. Our long-term goal is to develop postharvest biological control of brown rot originating from latent infection by $M$. fructicola occurring in the field, using naturally occurring antagonists. The ability to control latent infections may be different from protecting wounds, as the most critical part of the mechanism of biocontrol for latent infections is the direct colonization of the pathogen appressoria. Our current research is focused on developing methods for screening yeasts against latent infections, using both in vitro and in situ systems. 
Table 2. Identity of yeast isolates from nectarine fruit. Identifications are based on a BLAST search of GenBank using the DI/D2 sequences of the large subunit rRNA gene and, where available, confirmed with the ITS sequence

\begin{tabular}{|c|c|c|}
\hline NRRL' code & Kearneysville code & Isolate identification \\
\hline NRRL Y-48335 & Ne6T4-SII5 & Acremonium strictum or related species \\
\hline NRRL Y-48493 & Ne7TI-SIII & Aureobasidium pullulans ${ }^{2}$ \\
\hline NRRL Y-48494 & Ne7TI-SII7 & Aureobasidium pullulans \\
\hline NRRL Y-48496 & Ne7TI-SII2O & Aureobasidium pullulans \\
\hline NRRL Y-48498 & Ne7TI-S217 & Aureobasidium pullulans \\
\hline NRRL Y-48505 & $\mathrm{Ne} 7 \mathrm{TI}-\mathrm{S} 415$ & Aureobasidium pullulans \\
\hline NRRL Y-485 I0 & $\mathrm{Ne} 7 \mathrm{TI}-\mathrm{S} 515$ & Aureobasidium pullulans \\
\hline NRRL Y-48520 & $\mathrm{Ne} 7 \mathrm{~T} 2-\mathrm{S} 219$ & Aureobasidium pullulans \\
\hline NRRL Y-48524 & $\mathrm{Ne} 7 \mathrm{~T} 2-\mathrm{S} 315$ & Aureobasidium pullulans \\
\hline NRRL Y-48532 & $\mathrm{Ne} 7 \mathrm{~T} 2-\mathrm{S} 416$ & Aureobasidium pullulans \\
\hline NRRL Y-48542 & Ne7T2-S5II8 & Aureobasidium pullulans \\
\hline NRRL Y-48545 & Ne7T3-SII7 & Aureobasidium pullulans \\
\hline NRRL Y-48548 & Ne7T3-SIII Ib & Aureobasidium pullulans \\
\hline NRRL Y-48550 & Ne7T3-S2II & Aureobasidium pullulans \\
\hline NRRL Y-48554 & Ne7T3-S217 & Aureobasidium pullulans \\
\hline NRRL Y-48559 & Ne7T3-S312 & Aureobasidium pullulans \\
\hline NRRL Y-48567 & Ne7T3-S4I3 & Aureobasidium pullulans \\
\hline NRRL Y-48570 & Ne7T3-S517 & Aureobasidium pullulans \\
\hline NRRL Y-48579 & Ne7T4-SII9 & Aureobasidium pullulans \\
\hline NRRL Y-48587 & $\mathrm{Ne} 7 \mathrm{~T} 4-\mathrm{S} 214$ & Aureobasidium pullulans \\
\hline NRRL Y-48588 & $\mathrm{Ne} 7 \mathrm{~T} 4-\mathrm{S} 215$ & Aureobasidium pullulans \\
\hline NRRL Y-48594 & $\mathrm{Ne} 7 \mathrm{~T} 4-\mathrm{S} 318$ & Aureobasidium pullulans \\
\hline NRRL Y-48596 & Ne7T4-S4II & Aureobasidium pullulans \\
\hline NRRL Y-48599 & Ne7T4-S5I4 & Aureobasidium pullulans \\
\hline NRRL Y-48272 & Ne6TI-S214 & Aureobasidium pullulans \\
\hline NRRL Y-48273 & Ne6TI-S215 & Aureobasidium pullulans \\
\hline NRRL Y-48274 & Ne6TI-S4IIO & Aureobasidium pullulans \\
\hline NRRL Y-48276 & Ne6TI-S5I2 & Aureobasidium pullulans \\
\hline NRRL Y-48278 & Ne6TI-SII3 & Aureobasidium pullulans \\
\hline NRRL Y-48289 & Ne6TI-S514 & Aureobasidium pullulans \\
\hline NRRL Y-4829| & Ne6T2-SII8 & Aureobasidium pullulans \\
\hline NRRL Y-48292 & Ne6T2-SII9b & Aureobasidium pullulans \\
\hline NRRL Y-48293 & Ne6T2-SIIII & Aureobasidium pullulans \\
\hline NRRL Y-48298 & Ne6T2-S214 & Aureobasidium pullulans \\
\hline NRRL Y-48304 & Ne6T2-S313 & Aureobasidium pullulans \\
\hline NRRL Y-48307 & Ne6T2-S3IIO & Aureobasidium pullulans \\
\hline NRRL Y-483। I & Ne6T2-S4I2 & Aureobasidium pullulans \\
\hline NRRL Y-48319 & Ne6T2-S516b & Aureobasidium pullulans \\
\hline NRRL Y-48323 & Ne6T2-S5III & Aureobasidium pullulans \\
\hline NRRL Y-48327 & Ne6T3-SII9 & Aureobasidium pullulans \\
\hline NRRL Y-48329 & Ne6T3-S217 & Aureobasidium pullulans \\
\hline NRRL Y-4833। & Ne6T3-S317 & Aureobasidium pullulans \\
\hline NRRL Y-48334 & Ne6T3-S518 & Aureobasidium pullulans \\
\hline NRRL Y-48336 & Ne6T4-SII6 & Aureobasidium pullulans \\
\hline NRRL Y-485। 2 & Ne7TI-S519a & Auriculibuller fuscus \\
\hline NRRL Y-485। 4 & Ne7TI-S5II4 & Auriculibuller fuscus \\
\hline NRRL Y-48572 & Ne7T3-S5III & Auriculibuller fuscus \\
\hline NRRL Y-48340 & Ne6T4-S3IIO & Candida sp. nov. I \\
\hline NRRL Y-48342 & Ne6T4-S4I2 & Candida sp. nov. 2 \\
\hline NRRL Y-48543 & Ne7T3-SII3 & Crypt VKMY2958 \\
\hline NRRL Y-48544 & Ne7T3-SII5 & Crypt VKMY2958 \\
\hline NRRL Y-48546 & Ne7T3-SII8 & Crypt VKMY2958 \\
\hline NRRL Y-4859| & $\mathrm{Ne} 7 \mathrm{~T} 4-\mathrm{S} 313$ & Crypt VKMY2958 \\
\hline NRRL Y-48275 & Ne6TI-S4I5 & Cryptococcus magnus \\
\hline NRRL Y-483|4 & Ne6T3-S4I6 & Cryptococcus magnus \\
\hline NRRL Y-48317 & Ne6T2-S4III & Cryptococcus magnus \\
\hline
\end{tabular}


Table 2. Continued

\begin{tabular}{|c|c|c|}
\hline NRRL' code & Kearneysville code & Isolate identification \\
\hline NRRL Y-48539 & Ne7T2-S5III & Cryptococcus sp. nov. \\
\hline NRRL Y-48540 & Ne7T2-S5II3 & Cryptococcus sp. nov. \\
\hline NRRL Y-48566 & Ne7T3-S4IIa & Cryptococcus sp. nov. \\
\hline NRRL Y-48574 & Ne7T3-S5II3 & Cryptococcus sp. nov. \\
\hline NRRL Y-48590 & Ne7T4-S218 & Cryptococcus sp. nov. \\
\hline NRRL Y-48282 & Ne6TI-S2III & Cryptococcus sp. nov. \\
\hline NRRL Y-4830I & Ne6T2-S2III & Cryptococcus sp. nov. \\
\hline NRRL Y-483I2 & Ne6T2-S4I5 & Cryptococcus sp. nov. \\
\hline NRRL Y-48320 & Ne6T2-S5I8 & Cryptococcus sp. nov. \\
\hline NRRL Y-48326 & Ne6T3-SII8 & Cryptococcus sp. nov. \\
\hline NRRL Y-48553 & Ne7T3-S216 & Cryptococcus victoriae \\
\hline NRRL Y-48562 & Ne7T3-S318 & Cryptococcus victoriae \\
\hline NRRL Y-48497 & $\mathrm{Ne} 7 \mathrm{TI}-\mathrm{S} 214$ & Cryptococcus weiringae \\
\hline NRRL Y-48500 & Ne7TI-S2II 4 & Cryptococcus weiringae \\
\hline NRRL Y-48502 & Ne7TI-S3II4 & Cryptococcus weiringae \\
\hline NRRL Y-48506 & Ne7TI-S419b & Cryptococcus weiringae \\
\hline NRRL Y-48508 & Ne7TI-S4II 8 & Cryptococcus weiringae \\
\hline NRRL Y-485। 5 & Ne7T2-SIII & Cryptococcus weiringae \\
\hline NRRL Y-485। 6 & $\mathrm{Ne} 7 \mathrm{~T} 2-\mathrm{SI} / 2$ & Cryptococcus weiringae \\
\hline NRRL Y-485। 7 & $\mathrm{Ne} 7 \mathrm{~T} 2-\mathrm{S} 211$ & Cryptococcus weiringae \\
\hline NRRL Y-485। 8 & $\mathrm{Ne} 7 \mathrm{~T} 2-\mathrm{S} 2 \mathrm{I} 2$ & Cryptococcus weiringae \\
\hline NRRL Y-485। 9 & $\mathrm{Ne} 7 \mathrm{~T} 2-\mathrm{S} 213$ & Cryptococcus weiringae \\
\hline NRRL Y-4853। & $\mathrm{Ne} 7 \mathrm{~T} 2-\mathrm{S} 415$ & Cryptococcus weiringae \\
\hline NRRL Y-48533 & $\mathrm{Ne7T2-S4II2}$ & Cryptococcus weiringae \\
\hline NRRL Y-48536 & Ne7T2-S5IIa & Cryptococcus weiringae \\
\hline NRRL Y-48537 & Ne7T2-S514 & Cryptococcus weiringae \\
\hline NRRL Y-48577 & Ne7T4-SIII & Cryptococcus weiringae \\
\hline NRRL Y-48584 & Ne7T4-S2II & Cryptococcus weiringae \\
\hline NRRL Y-48585 & $\mathrm{Ne} 7 \mathrm{~T} 4-\mathrm{S} 212$ & Cryptococcus weiringae \\
\hline NRRL Y-4827I & Ne6TI-SII2 & Cryptococcus wieringae \\
\hline NRRL Y-48277 & Ne6TI-S5I3 & Cryptococcus wieringae \\
\hline NRRL Y-48297 & $\mathrm{Ne} 6 \mathrm{~T} 2-\mathrm{S} 213$ & Cryptococcus wieringae \\
\hline NRRL Y-48306 & Ne6T2-S319 & Cryptococcus wieringae \\
\hline NRRL Y-48324 & Ne6T3-SIII & Cryptococcus wieringae \\
\hline NRRL Y-48325 & Ne6T3-SII3 & Cryptococcus wieringae \\
\hline NRRL Y-48557 & Ne7T3-S2II3 & Cryptococcus sp. nov. \\
\hline NRRL Y-48563 & Ne7T3-S3IIO & Cryptococcus sp. nov. \\
\hline NRRL Y-48575 & Ne7T3-S5II7 & Cryptococcus sp. nov. \\
\hline NRRL Y-48583 & Ne7T4-SIII7 & Cryptococcus sp. nov. \\
\hline NRRL Y-48595 & Ne7T4-S319 & Cryptococcus sp. nov. \\
\hline NRRL Y-4860। & Ne7T4-S5II2 & Cryptococcus sp. nov. \\
\hline NRRL Y-48560 & Ne7T3-S314 & Dothichiza pithyophila \\
\hline NRRL Y-48279 & Ne6TI-SII4 & Erythrobasidium hasegawianum \\
\hline NRRL Y-48295 & Ne6T2-SIII4 & Erythrobasidium hasegawianum \\
\hline NRRL Y-48300 & Ne6T2-S219 & Erythrobasidium hasegawianum \\
\hline NRRL Y-48305 & Ne6T2-S3I5b & Erythrobasidium hasegawianum \\
\hline NRRL Y-48310 & Ne6T2-S4II & Erythrobasidium hasegawianum \\
\hline NRRL Y-48338 & Ne6T4-S215 & Hanseniaspora uvarum \\
\hline NRRL Y-48589 & $\mathrm{Ne} 7 \mathrm{~T} 4-\mathrm{S} 217$ & Metschnikowia fructicola/pulcherrima \\
\hline NRRL Y-48529 & $\mathrm{Ne} 7 \mathrm{~T} 2-\mathrm{S} 413$ & near Metschnikowia kunwiensis \\
\hline NRRL Y-48507 & Ne7TI-S4II7 & near Pseudeurotium zonatim \\
\hline NRRL Y-48534 & Ne7T2-S4II4 & near Rhodotorula philya \\
\hline NRRL Y-48576 & Ne7T3-S5II8 & near Rhodotorula philya \\
\hline NRRL Y-48344 & Ne6T4-S4I7 & Pichia Kluyveri \\
\hline NRRL Y-48549 & Ne7T3-SIII2 & Rhodotorula philya \\
\hline NRRL Y-48287 & Ne6TI-S4I6a & Rhodotorula glutinis \\
\hline
\end{tabular}


Table 2. Continued

\begin{tabular}{|c|c|c|}
\hline NRRL' code & Kearneysville code & Isolate identification \\
\hline NRRL Y-48290 & Ne6T2-SII4 & Rhodotorula glutinis \\
\hline NRRL Y-48308 & Ne6T2-S3II2 & Rhodotorula glutinis \\
\hline NRRL Y-48328 & Ne6T3-S214 & Rhodotorula glutinis \\
\hline NRRL Y-48495 & Ne7TI-SIII7 & Rhodotorula hinnulea \\
\hline NRRL Y-4850। & Ne7TI-S21I 8 & Rhodotorula hinnulea \\
\hline NRRL Y-48525 & Ne7T2-S317 & Rhodotorula hinnulea \\
\hline NRRL Y-48527 & Ne7T2-S3II 5 & Rhodotorula hinnulea \\
\hline NRRL Y-48528 & Ne7T2-S31I 6 & Rhodotorula hinnulea \\
\hline NRRL Y-48535 & Ne7T2-S4II 5 & Rhodotorula hinnulea \\
\hline NRRL Y-4854 I & Ne7T2-S5II7 & Rhodotorula hinnulea \\
\hline NRRL Y-48556 & Ne7T3-S2III & Rhodotorula hinnulea \\
\hline NRRL Y-4856I & Ne7T3-S316b & Rhodotorula hinnulea \\
\hline NRRL Y-48565 & Ne7T3-S3II2 & Rhodotorula hinnulea \\
\hline NRRL Y-48569 & Ne7T3-S4III & Rhodotorula hinnulea \\
\hline NRRL Y-48573 & Ne7T3-S5II2 & Rhodotorula hinnulea \\
\hline NRRL Y-48580 & Ne7T4-SIII4 & Rhodotorula hinnulea \\
\hline NRRL Y-48582 & Ne7T4-SIII6 & Rhodotorula hinnulea \\
\hline NRRL Y-48597 & Ne7T4-S4II 2 & Rhodotorula hinnulea \\
\hline NRRL Y-48280 & Ne6TI-S2I2 & Rhodotorula hinnulea or undescribed sister species \\
\hline NRRL Y-48284 & Ne6TI-S315 & Rhodotorula hinnulea or undescribed sister species \\
\hline NRRL Y-48309 & Ne6T2-S3II4 & Rhodotorula hinnulea or undescribed sister species \\
\hline NRRL Y-48322 & Ne6T2-S5IIO & Rhodotorula hinnulea or undescribed sister species \\
\hline NRRL Y-48503 & Ne7TI-S3II7 & Rhodotorula pinicola \\
\hline NRRL Y-485 I 3 & $\mathrm{Ne7TI-S5II2}$ & Rhodotorula pinicola \\
\hline NRRL Y-48522 & Ne7T2-S2II4 & Rhodotorula pinicola \\
\hline NRRL Y-48558 & Ne7T3-S2II5 & Rhodotorula pinicola \\
\hline NRRL Y-4858। & Ne7T4-SIII5 & Rhodotorula pinicola \\
\hline NRRL Y-4828। & Ne6TI-S2IIO & Rhodotorula sp. near Rhodotorula laryngis \\
\hline NRRL Y-48283 & Ne6TI-S314 & Rhodotorula sp. near Rhodotorula laryngis \\
\hline NRRL Y-48286 & Ne6TI-S4I3 & Rhodotorula sp. near Rhodotorula laryngis \\
\hline NRRL Y-48288 & Ne6TI-S4I8 & Rhodotorula sp. near Rhodotorula laryngis \\
\hline NRRL Y-48296 & Ne6T2-SIII5 & Rhodotorula sp. near Rhodotorula laryngis \\
\hline NRRL Y-48302 & Ne6T2-S2II2 & Rhodotorula sp. near Rhodotorula laryngis \\
\hline NRRL Y-483। 5 & Ne6T3-S4I7 & Rhodotorula sp. near Rhodotorula laryngis \\
\hline NRRL Y-483 | 6 & Ne6T2-S4IIO & Rhodotorula sp. near Rhodotorula laryngis \\
\hline NRRL Y-48332 & Ne6T3-S3III & Rhodotorula sp. near Rhodotorula laryngis \\
\hline NRRL Y-48337 & Ne6T4-S213 & Rhodotorula sp. near Rhodotorula laryngis \\
\hline NRRL Y-48499 & Ne7TI-S2IIO & Sporidiobolus pararoseus \\
\hline NRRL Y-48509 & Ne7TI-S5l4 & Sporidiobolus pararoseus \\
\hline NRRL Y-485 I | & Ne7TI-S5I8b & Sporidiobolus pararoseus \\
\hline NRRL Y-4852I & Ne7T2-S2II0 & Sporidiobolus pararoseus \\
\hline NRRL Y-48530 & Ne7T2-S4I4 & Sporidiobolus pararoseus \\
\hline NRRL Y-48538 & Ne7T2-S5I5 & Sporidiobolus pararoseus \\
\hline NRRL Y-48547 & Ne7T3-S IIIIa & Sporidiobolus pararoseus \\
\hline NRRL Y-4855 | & Ne7T3-S213 & Sporidiobolus pararoseus \\
\hline NRRL Y-48568 & Ne7T3-S4I4a & Sporidiobolus pararoseus \\
\hline NRRL Y-4857I & Ne7T3-S519b & Sporidiobolus pararoseus \\
\hline NRRL Y-48586 & Ne7T4-S213 & Sporidiobolus pararoseus \\
\hline NRRL Y-48592 & Ne7T4-S316 & Sporidiobolus pararoseus \\
\hline NRRL Y-48593 & Ne7T4-S317 & Sporidiobolus pararoseus \\
\hline NRRL Y-48598 & Ne7T4-S513 & Sporidiobolus pararoseus \\
\hline NRRL Y-48600 & Ne7T4-S516 & Sporidiobolus pararoseus \\
\hline NRRL Y-48299 & Ne6T2-S218 & Sporidiobolus pararoseus_-probable undescribed sister species \\
\hline NRRL Y-48303 & Ne6T2-S3II & Sporidiobolus pararoseus - probable undescribed sister species \\
\hline NRRL Y-48313 & Ne6T3-S3110 & Sporidiobolus pararoseus - probable undescribed sister species \\
\hline
\end{tabular}


Table 2. Continued

\begin{tabular}{lll}
\hline NRRL' code & Kearneysville code & \multicolumn{1}{c}{ Isolate identification } \\
\hline NRRL Y-483I8 & Ne6T2-S516a & Sporidiobolus pararoseus-probable undescribed sister species \\
NRRL Y-48330 & Ne6T3-S2II0 & Sporidiobolus pararoseus-probable undescribed sister species \\
NRRL Y-48333 & Ne6T3-S418 & Sporidiobolus pararoseus-probable undescribed sister species \\
NRRL Y-48339 & Ne6T4-S316 & Sporidiobolus pararoseus-probable undescribed sister species \\
NRRL Y-48354 & Ne6T2-SIII2 & Sporidiobolus pararoseus-probable undescribed sister species \\
NRRL Y-48355 & Ne6T3-S318 & Sporidiobolus pararoseus-probable undescribed sister species \\
NRRL Y-48555 & Ne7T3-S218 & Sporobolomyces phaffi \\
NRRL Y-48552 & Ne7T3-S215 & Sporobolomyces roseus \\
NRRL Y-48504 & Ne7TI-S3120 & Taphrina carnea \\
NRRL Y-48564 & Ne7T3-S31II & Taphrina carnea \\
NRRL Y-48523 & Ne7T2-S21I7 & Taphrina deformans \\
NRRL Y-48578 & Ne7T4-SI6 & Taphrina deformans \\
NRRL Y-48294 & Ne6T2-SIII3 & Tremella globispora or a sister species \\
NRRL Y-48343 & Ne6T4-S416 & Zygosaccharomyces bailii \\
\hline
\end{tabular}

I National Center for Agricultural Utilization Research (ARS Culture Collection, Pioria, IL, USA).

2 Differed by two Ns and three indels in LSU DI/D2 from reference AFTOL-ID9 I2, GenBank DQ470956. 0 diff. in ITS with EF690466. There is no living ex-type for A. pullulans, so the interpretation is that the following strains are A. pullulans or a closely related species.

Table 3. Bioconrol potential of yeasts isolated from nectarine fruit at various times against wound infection by Monilinia fructicola causing brown rot of stone fruits

\begin{tabular}{lc}
\hline $\begin{array}{l}\text { Biocontrol category } \\
\text { (\% wounds infected) }\end{array}$ & $\begin{array}{c}\text { Yeasts in each category } \\
\text { (\%) }\end{array}$ \\
\hline 0 & 2.2 \\
$<34$ & 23.0 \\
$34-66$ & 36.3 \\
$66-100$ & 38.5 \\
\hline
\end{tabular}

${ }^{\prime}$ Results from screening on the fruit inoculated with the highest $\left(10^{5}\right.$ conidia/ml) concentration of $M$. fructicola.

\section{Acknowledgements}

We would like to thank Roger Lewis and Eleanor BasehoarPowers for their technical assistance in this project.

\section{References}

Beech FW. 1993. Yeast in cider-making. In The Yeast, vol 5, Rose AH, Harrison JS (eds). Academic Press: London; $169-213$.

Blakeman JP. 1985. Ecological succession of leaf surface microorganisms in relation to biological control. In Biological Control on the Phylloplane, Windels CE, Lindow SE (eds). APS Press: St. Paul, MN; 6-30.

Buhagiar RWM, Barnett JA. 1971. The yeast of strawberries. J Appl Bact 34: 727-739.

Calvente V, de Orellano ME, Sansone G, et al. 2001. Effect of nitrogen source and $\mathrm{pH}$ on siderophore production by Rhodotorula strains and their application to biocontrol of phytopathogenic moulds. J Ind Microbiol Biotechnol 26: $226-229$.
Clark DS, Wallace RH, David JJ. 1954. Yeast occurring on apples and in apple cider. Can J Microbiol 1: 145-149.

Chand-Goyal T, Spotts RA. 1996. Enumeration of bacterial and yeast colonists of apple fruits and identification of epiphytic yeasts on pear fruits in the Pacific Northwest United States. Microbiol Res 151: 427-432.

Dimakopoulou M, Tjamos SE, Antoniou PP, et al. 2008. Phyllosphere grapevine yeast Aureobasidium pullulans reduces Aspergillus carbonarius (sour rot) incidence in wine-producing vineyards in Greece. Biol Control 46: 158-165.

Davenport RR. 1976a. Ecological concepts in studies of microorganisms on areal plant surfaces. In Microbiology of Plant Surfaces, Dickinson CH, Preece TE (eds). Academic Press: New York, 199-215.

Davenport RR. 1976b. Distribution of yeast and yeast-like organisms from aerial surfaces of developing apples and grapes. In Microbiology of Plant Surfaces, Dickinson CH, Preece TE (eds). Academic Press: London, 325-359.

Dennis C. 1976. The microflora of the surface of soft fruits. In Microbiology of Aerial Plant Surfaces, Dickinson CH, Preece TF (eds). Academic Press: London; 419-432.

Droby S, Lischinski S, Cohen L, et al. 1999. Characterization of an epiphytic yeast population of grapefruit capable of suppression of green mold decay caused by Penicillium digitatum. Biol Control 16: 27-34.

Ippolito A, Nigro F. 2000. Impact of preharvest application of biological control agents on postharvest diseases of fresh fruits and vegetables. Crop Protect 19: 715-723.

Janisiewicz WJ. 1987. Postharvest biological control of blue mold of apples. Phytopathology 77: 481-485.

Janisiewicz WJ, Bors RH. 1995. Development of microbial community of bacterial and yeast antagonists to control woundinvading postharvest pathogens of fruits. Appl Environ Microbiol 61: $3261-3267$.

Janisiewicz WJ, Buyer JS. 2010. Culturable bacterial microflora associated with nectarine fruit and their potential for control of brown rot. Can J Microbiol. 65: (in press). 
Janisiewicz WJ, Korsten L. 2002. Biological control of postharvest diseases of fruits. Annu Rev Phytopathol 40: 411-441. DOI:10.1146/annurev.phyto.40.120401.130158.

Janisiewicz WJ, Marchi A. 1992. Control of storage rots on various pear cultivars with saprophytic strain of Pseudomonas syringae. Plant Dis 76: 555-560.

Janisiewicz WJ, Tworkoski TJ, Kurtzman CP. 2001. Biocontrol potential of Metschnikowia pulcherrima strains against blue mold of apple. Phytopathology 91: 1098-1108.

Kunz S. 2004. Development of 'Blossom-Protect' - a yeast preparation for the reduction of blossom infections by fire blight. In 11th International Conference on Cultivation Technique and Phytopathological Problems in Organic Fruit-Growing and Viticulture, Boos M (ed.). Fördergemeinschaft Ökologischer Obstbau: Weinsberg; 108-114.

Kurtzman CP, Robnett CJ. 1998. Identification and phylogeny of ascomycetous yeasts from analysis of nuclear large subunit (26S) ribosomal DNA partial sequences. Antonie van Leeuwenhoek 73: 331-371.

Kurtzman CP, Robnett CJ. 2003. Phylogenetic relationships among yeasts of the 'Saccharomyces complex' determined from multigene sequence analyses. FEMS Yeast Res 3: 417-432.

Leverentz B, Conway WS, Janisiewicz WJ, et al. 2006. Biocontrol of the foodborne pathogens Listeria monocytogenes and Salmonella poona on fresh-cut apples with naturally occurring bacterial and yeast antagonist. Appl Environ Microbiol 72: $1135-1140$.

Lebinger W, Breuker B, Hahn M, Mendgen K. 1997. Control of postharvest pathogens and colonization of the apple surface by antagonistic microorganisms in the field. Phytopathology 87: $1103-1110$.

Lima G, De Curtis F, Castoria R, De Cicco V. 1998. Activity of the yeasts Cryptococcus laurentii and Rhodotorula glutinis against post-harvest rots on different fruits. Biocontrol Sci Technol 8: 257-267.

Lima G, Ippolito A, Nigro F, Salerno M. 1997. Effectiveness of Aureobasidium pullulans and Candida oleophila against postharvest strawberry rots. Postharvest Biol Technol 10: 169-178.

McCune B, Grace JB. 2002. Analysis of Ecological Communities. MjM Software Design: Gleneden Beach, OR; 300 pp.

Roberts RG. 1990. Postharvest biological control of gray mold of apple by Cryptococcus laurentii. Phytopathology 80: 526-530.

Sasaki Y, Yoshida T. 1959. Distribution and classification studies on the wild yeasts or budding fungi on the fresh fruit in Hokkaido. J Facul Agric Hokkaido Univ Sapporo 51(1): 194-220.

Singh JP, Kainsa RL. 1983. Microbial flora of grapes in relation to storage and spoilage. Indian Phytopathol 36: 72-76.

Stalarova V. 1982. The presence of yeast and yeast-like microorganisms on cherry fruits (Cerasus avium L. Moench). Biolǒgia (Bratislava) 37: 1115-1120.

Stalarova V. 1984. Share of the species Hanseniaspora uvarum in natural population of yeasts. Biológia (Bratislava) 39: 313-319.

Usall J, Teixidó N, Fons E, Viñas I. 2000. Biological control of blue mould on apple by a strain of Candida sake under several controlled atmosphere conditions. Int J Food Microbiol 58: $83-92$.

Wadia KD, Manohorachary C, Janaki CH. 1983. Fruit surface mycoflora of Vitis vinifera L. and Capsicum annum L. in relation to their fruit rot diseases. Proc Indian Nat Sci Acad B46: $371-379$.

Williams AJ, Wallace RH, Clark DS. 1956. Changes in the yeast populations on Quebec apples during ripening. Can J Microb 2: $45-648$.

Wilson CL, Wisniewski ME, Droby E, Chalutz E. 1993. A selection strategy for microbial antagonists to control postharvest diseases of fruits and vegetables. Sci Hort 53: $183-189$.

Zheng XD, Zhang HY, Sun P. 2005. Biological control of postharvest green mold decay on oranges by Rhodotorula glutinis. European Food Res Technol 220: 353-357. 